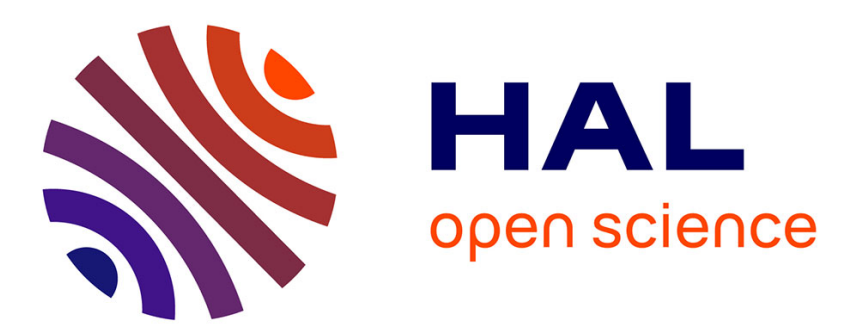

\title{
Solveur de type Godunov pour simuler les écoulements turbulents compressibles
}

\author{
Alain Forestier, Jean-Marc Hérard, Xavier Louis
}

\section{To cite this version:}

Alain Forestier, Jean-Marc Hérard, Xavier Louis. Solveur de type Godunov pour simuler les écoulements turbulents compressibles. Comptes Rendus de l'Académie des Sciences - Series I - Mathematics, 1997, 324 (8), pp.919-926. 10.1016/S0764-4442(97)86969-8 . hal-01579899

\section{HAL Id: hal-01579899 \\ https://hal.science/hal-01579899}

Submitted on 8 Jan 2020

HAL is a multi-disciplinary open access archive for the deposit and dissemination of scientific research documents, whether they are published or not. The documents may come from teaching and research institutions in France or abroad, or from public or private research centers.
L'archive ouverte pluridisciplinaire HAL, est destinée au dépôt et à la diffusion de documents scientifiques de niveau recherche, publiés ou non, émanant des établissements d'enseignement et de recherche français ou étrangers, des laboratoires publics ou privés. 
archives-ouvertes

\title{
Solveur de type Godunov pour simuler les écoulements turbulents compressibles
}

\author{
Alain Forestier, Jean-Marc Hérard, Xavier Louis
}

\section{To cite this version:}

Alain Forestier, Jean-Marc Hérard, Xavier Louis. Solveur de type Godunov pour simuler les écoulements turbulents compressibles. Comptes Rendus de l'Académie des Sciences - Series I - Mathematics, Elsevier, 1997, 324 (8), pp.919-926. hal-01579899

\section{HAL Id: hal-01579899 \\ https://hal.archives-ouvertes.fr/hal-01579899}

Submitted on 8 Jan 2020

HAL is a multi-disciplinary open access archive for the deposit and dissemination of scientific research documents, whether they are published or not. The documents may come from teaching and research institutions in France or abroad, or from public or private research centers.
L'archive ouverte pluridisciplinaire HAL, est destinée au dépôt et à la diffusion de documents scientifiques de niveau recherche, publiés ou non, émanant des établissements d'enseignement et de recherche français ou étrangers, des laboratoires publics ou privés. 


\title{
Solveur de type Godunov pour simuler les écoulements turbulents compressibles
}

\author{
Alain FORESTIER, Jean-Mare IÉRARD et Xavier LOUIS \\ A. F. et X. L. : CEA Cadlarache, DER/SERALTME, 13108 Suint-1'aul-lrz-Duranien
}

J.-M. II. : EDF, Jirection Étuden et Rerherrhes,

Départensent Lalooratoine National d'Ilyclrualicape, 6, quai Watior. 78410 Chatou.

Résumé. On présente ici un solveur de type Godunov qui permet la simulation d'un système hyperbolique non conservatif sur des maillages non structurés. Le système provient du modèle de turbulence compressible $K^{\prime}-\varepsilon$. On présente l'inégalité d'entropie associće au système considéré et la solution du problème de Riemann unidimensionnel obtenue ẹn connectant les états par des relations de saut approchées à travers les discontinuités.

\section{A Godunov type solver for turbulent compressible flots}

\begin{abstract}
We present here a Godunov npe solver which enables us to compute a non consenvative hyperbolic sistiem on unstructured meshes. The convective system comes from the standard turbulent compressible $K^{\circ}-\varepsilon$ model. An entropy. inceuulity is given first: then the solution of the associated one dimenssional Riemumn powblem is given. using

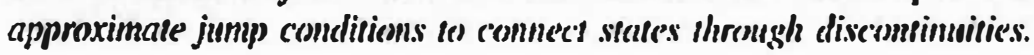

\section{. AbridgedEnglish Version}

We examine the non conservative hyperbolic system arising from the $K-\varepsilon$ turbulent compressible model (see Equations (1)-(12), numbers refer to the French version) arising from Favre's averaging technique. Variables $\rho, U, P$, and $E$ denote respectively the mean density, mean velocity, mean pressure, and mean total energy; $K=\overline{p m u_{1}^{\prime \prime} u_{1}^{\prime \prime}} / 2$ and $\varepsilon$ represent the turbulent kinetic energy and the turbulent dissipation. The state law is given by (9). We set $\mathbf{Z}^{t}=(\rho, \rho U . \rho V, \rho U, E, K, \varepsilon)$ and get an entropy inequality (see proposition II). We also set $T=\rho^{-1} P, S_{1,}=U_{1,3}+U_{J, 2}$; the entropy-entropy flux pair $\left(\eta(\mathbf{Z}), f_{\eta}^{i}(\mathbf{Z})\right)$ is given by (13). Governing equations of the non conservative system are ((17)-(21)). The 1-wave and 5-wave are GNL fields. Other waves are Linearly Degenerate. Riemann invariants are detailed in (22)-(26) (setting: $\eta=1+\frac{2}{3} C_{\varepsilon_{1}}$ and $\left.s=P p^{-1}\right)$. We introduce standard notations: $\bar{\phi}=\left(\phi_{G}+\phi_{D}\right) / 2_{1}[\phi]=\phi_{D}-\phi_{G}$. where $\phi_{G}$ et $\phi_{D}$ represent the left and right states on each side of a travelling discontinuity. We consider approximate jump conditions ((27)-(28)) ( $\sigma$ denotes the speed of the discontinuity). It may be shown that rarefaction curves and shock curves (see (30)-(31)) 
are $C^{2}$ connected (see [12]). Even more, the entropy inequality (29) enables to check that $[U]<0$ through shocks. Thus, we may examine the Cauchy problem arising from (17)-(21) and the initinl data:

$$
\begin{aligned}
& W(x<0, t=0)=W_{l}=\left(\rho_{L}, \rho_{L} U_{L}, E_{L}, K_{L}, \varepsilon_{L}\right), \\
& \mathbf{W}(x>0, t=0)=W_{R}=\left(\rho_{R}, \rho_{R} U_{R}, E_{R}, K_{R}, \varepsilon_{R}\right) .
\end{aligned}
$$

Propostrion. - The one dimensional Riemann problem associated with (17)-(21) and jump conditions (27-28) admits a unique entropy-consistent solution with positive density provided that:

$$
U_{R}-U_{L}<X_{L}+X_{R}
$$

setting $c^{2}=\gamma P / \rho+10 K /(9 \rho)$ and

$$
X_{i}=\int_{0}^{\rho_{1}} \frac{c^{\prime}\left(\rho, B_{1} K \rho^{-5 / 3}\right)}{\rho} d \rho \quad \text { with } i=L \text { or } R .
$$

The mean pressure $P$ and the turbulent kinetic energy $K$ remain positive in the $(x, t)$ plane.

A numerical Godunov-type scheme was implemented in a two-dimensional framework, on unstructured meshes. The one-dimensional Finite-Volume scheme is detailed in (33)-(38). A non conservative version of the Roe-type scheme has also been defined in [7] and compared with the Godunov-type scheme. A numerical computation is displayed in figure 1.

\section{Introduction}

On s'intéresse à la modélisation numérique des écoulements turbulents compressibles à forte dominante convective, en simulant le système de turbulence compressible à deux équations, appelé modèle $K-\varepsilon$ (voir [1], [2] et [3]), qui est basé sur une formulation en moyenne de Favre (voir [4]). Dans le cadre de la méthode des Eléments Finis, une proposition a été faite dans [5] pour aborder İa résolution des modéles de turbulence compressible à une ou deux équations. Dans ie cadre des méthodes de Volumes Finis, deux propositions distinctes ont été formulées dans [2] et [6], la dernièré étant basée sur la similitude du pseuda-système convectif associé au modẻle $K-\epsilon$ et du système des équations d'Euler multi-espèces (voir également [3]). On s'intéresse ici à l'analyse et au traitement numérique du système complet de convection associé à ce système qui est non conservatif, et on donne la solution du problème de Riemann, pour un choix de relations de saut approchées. Un résultat numérique de tube à choc par un schéma de type Godunov est présenté.

\section{Le modèle de turbulence compressible}

Le modèle continu choisi pour décrire les évolutions du fluide monophasique en état d'agitation turbulente est le système à deux équations $K-\varepsilon$. Il est présenté ici en introduisant l'énergie cinétique turbulente $K=\overline{\rho \tau u_{\imath}^{\prime \prime} u_{\imath}^{\prime \prime}} / 2$, la densité moyenne $\rho$, la vitesse moyenne $U$. l'énergie totale moyenne $E$ et la viscosité turbulente $\mu_{\ell}$ :

$$
\begin{aligned}
& (\rho)_{, 8}+\left(\rho U_{8}\right)_{18}=0 \\
& \left(\rho U_{i}\right)_{, 2}+\left(\rho U_{i} U_{j}+\left(P+\frac{2 K}{3}\right) \delta_{i j}\right)_{, j}=-\left(\tau_{i j}^{u}\right)_{, j} \\
& (E)_{, \ell}+\left(U_{j} E\right)_{, j}+\left(U_{1}\left(P+\frac{2 K}{3}\right) \delta_{i j}\right)_{j}=-\left(U_{v}+{ }_{i j}^{\prime \prime}\right)_{, j}-\left(\Phi_{j}^{E}\right)_{j},
\end{aligned}
$$




$$
(K)_{, i}+\left(K U_{i}\right)_{, i}+\frac{2}{3} K\left(U_{i}\right)_{, i}=S_{k}-\left(\phi_{i}^{K}\right)_{, i},
$$

en considérant les relations de fermeture :

$$
\tau_{1 j}^{*}=-\mu_{\mathrm{eff}}\left(U_{i, j}+U_{3, i}-\frac{2}{3} U_{1,1} \delta_{i j}\right)
$$

$$
\mu_{\mathrm{eff}}=\mu_{\mathrm{lam}}+\mu^{t} \equiv \mu_{\mathrm{lam}}+C_{\mu} \frac{K^{2}}{\varepsilon}
$$

$$
S_{K}=\frac{\mu^{2}}{2}\left(U_{i, j}+U_{j, i}-\frac{2}{3} U_{1,1} \delta_{i j}\right)\left(U_{i, j}+U_{j, 2}\right)-\epsilon \equiv \pi-\varepsilon,
$$

$$
\phi_{i}^{K}=-\sigma_{K}\left(\rho^{-1} K\right)_{, 2} ; \quad \phi_{z}^{E}=\phi_{z}^{K}-\sigma_{E}\left(\rho^{-1} P\right)_{, 2},
$$

(voir [1], [2], [3] et [5]). La loi d'état moyennée des gaz parfaits ( $\mathrm{P}$ désigne la pression moyenne) est :

$$
P=(\gamma-1)\left(E-\frac{1}{2} \rho U_{j} U_{j}-K\right) \text {. }
$$

La dissipation visqueuse $\epsilon$ associée au mouvement fluctuant est solution de :

$$
(\varepsilon)_{, t}+\left(\varepsilon U_{i}\right)_{, i}+\frac{2}{3} C_{\varepsilon 1} \varepsilon\left(U_{i}\right)_{, i}=S_{\varepsilon}-\left(\phi_{1}^{\varepsilon}\right)_{, 2} .
$$

Le teme source $S E$ et le fiux diffusif turbulent associés s’écrivent :

$$
S_{\varepsilon}=\frac{\varepsilon}{K}\left(C_{\varepsilon 1} \pi-C_{\varepsilon 2} \varepsilon\right) ; \quad \phi_{i}^{\varepsilon}=-\sigma_{\varepsilon}\left(\rho^{-1} \varepsilon\right)_{, i} .
$$

Les valeurs classiques, issues de la modélisation en situation incompressible, sont :

$$
C_{\varepsilon 1}=1.44 ; \quad C_{\varepsilon 2}=1.92 ; \quad C_{\mu}=0.09
$$

Les fonctions $\sigma_{E}, \sigma_{K}, \sigma_{\varepsilon}$ doivent être positives.

\section{Caractérisation entropique}

On pose : $\mathbf{Z}^{\ell}=(\rho, \rho U, \rho V, \rho \zeta, E, K, \varepsilon)$ et on introduit le couple (entropie, flux d'entropie) :

$$
\eta(\mathbf{Z})=-\rho \log \left(P \rho^{-\gamma}\right), \quad f_{\eta}^{i}(\mathbf{Z})=U_{i} \eta .
$$

On a alors (voir [7]), pour toute solution régulière $Z$ de (1), (2), (3), (4), (9), la proposition suivante :

Proposition II. - Le système (1)-(12) admet l'inégalité d'entropie suivante :

$$
\begin{aligned}
& (\eta(Z))_{, \imath}+\left(f_{\eta}^{i}(Z)\right)_{, i}+\left((\gamma-1) \frac{\sigma_{E}}{T} T_{, 1}\right)_{, i} \\
& \quad=-(\gamma-1) \frac{\sigma_{E}}{T^{2}} T_{, i} T_{, i}-\frac{(\gamma-1)}{T}\left(\varepsilon+\frac{\mu_{\text {am }}}{2} S_{i j}\left(S_{i j}-\frac{1}{3} S_{11} \delta_{i j}\right)\right) \leq 0 .
\end{aligned}
$$




\section{A. Forestier, J.-M. Hérard et X. Louis}

On a noté ici : $T=\rho^{-1} P$ et $S_{\imath \jmath}=U_{\imath, \jmath}+U_{, 1 .}$. La caractérisation entropique est très proche de celle de la dynamique des gaz dans le cadre laminaire. Elle fait apparaitre le fait que la fonction de dissipation d'entropie est (explicitement) indépendante de la viscosité turbulente $\mu_{t}$. L'entropie est une fonction convexe (non stricte) de $Z$; elle permettra de sélectionner la solution admissible du problème de Riemann associé au système de convection non conservatif dans les champs VNL.

\section{Hyperbolicité du système de convection}

Le système de convection associé au membre de gauche du système ((1)-(4),(9)-(10)) est non conservatif et peut s'écrire dans les zones régulières (en notant : $\left.\mathbf{Z}^{t}=(\rho, \rho U, \rho V, \rho \mathrm{I} V, E, K, \varepsilon)\right)$ :

$$
\mathrm{Z}_{. \ell}+\mathbf{A}_{\mathrm{s}}(\mathbf{Z}) \mathbf{Z}_{. \imath}=\mathbf{0}
$$

On introduit alors une normale unitaire quelconque : $n^{t}=\left(n_{x}, n_{y}, n_{z}\right)$, et les notations suivantes :

$$
\mathcal{U}=n_{x} U+n_{y} V+n_{z} W . \quad r^{2}=\gamma P / \rho+10 K /(9 \rho) .
$$

Propostrion III. - Le système de convection non consenvatif (14) est hyperbolique non strict. Les valeurs propres s'écrivent dans le cas tridimensionnel :

$$
\lambda_{1}=\mathcal{U}-c^{\prime}, \quad \lambda_{1}=\mathcal{U} \quad(i=2,3,4,5,6) . \quad \lambda_{7}=\mathcal{U}+c^{\prime} .
$$

L'hyperbolicité du système est associée au respect de la positivité des densité et pression moyennes, et de l'énergie turbulente. Le système est invariant par rotation. On se restreint au cadre unidimensionnel :

$$
(\rho)_{. f}+(\rho U)_{. s}=0
$$

$$
(\rho U)_{, t}+\left(\rho U^{2}\right)_{. x}+P_{. s}+\frac{2}{3} K_{. x}=0 .
$$

$$
(E)_{. t}+(U(E+P+2 K / 3))_{. s}=0,
$$

La loi d'état est toujours (9) et on introduit la variable réduite : $W^{t}=(\rho, \rho U, E, K, \varepsilon)$. Les valeurs propres du système (17) à (2I) sont : $\lambda_{1}=U-r^{\prime}: \lambda_{t}=U(i=2,3,4): \lambda_{5}=U+c^{\prime}$. Les 1-onde et 5-onde sont VNL; le champ associé à la valeur propre triple /l est Linéairement Dégénéré (LD). Les invariants de Riemann sont (en posant : $\eta=1+\frac{2}{3} C_{\ell_{1}}$ et également $\left.s=\Gamma \rho^{-\gamma}\right)$ :

$$
\phi_{1}^{1,2,3,4}(W)=\left(4 . K \rho^{-5 / 3} \cdot l i+\int \frac{c^{\prime}\left(\rho \cdot s, k \rho^{-5 / 3}\right)}{\rho} d p \cdot \varepsilon \rho^{-\eta}\right)
$$




$$
\begin{gathered}
\phi_{2}^{1,2,3,4}(\mathrm{~W})=\left(U, P, P+\frac{2}{3} K, \varepsilon\right), \\
\phi_{3}^{1,2,3,4}(\mathrm{~W})=\left(U, s, P+\frac{2}{3} K, \varepsilon\right), \\
\phi_{3}^{1,2,3,4}(\mathrm{~W})=\left(U, s, P+\frac{2}{3} K, K\right), \\
\phi_{5}^{1,2,3,4}(\mathrm{~W})=\left(s, K \rho^{-5 / 3}, U \int \frac{c^{\prime}\left(\rho . s, K \rho^{-5 / 3}\right)}{\rho} d \rho, \varepsilon \rho^{-4}\right) .
\end{gathered}
$$

\section{Solution du problème de Riemann unidimensionnel}

On s'intéresse maintenant aux solutions discontinues du système (17)-(21) en se restreignant au cadre des ondes de choc de faible amplitude. On introduit les notations : $\bar{\phi}=\left(\phi_{G_{i}}+\phi_{D}\right) / 2,[\phi]=\phi_{D}-\phi_{G_{G}}$. où $\phi_{G}$ et $\phi_{D}$ désignent les valeurs gauche et droite de la discontinuité se déplaçant à la vitesse $\sigma$. On envisage les relations de saut approchées (voir [8], [9], [10], [1]] et [12]) :

$$
\begin{aligned}
& -\sigma[K]+[U K]+\frac{2}{3} \bar{K}[U]=0 \\
& -\sigma[\varepsilon]+[U \varepsilon]+\frac{2}{3} C_{\varepsilon 1} \bar{\varepsilon}[U]=0 .
\end{aligned}
$$

Ces relations de saut permettent ici d'obtenir une connection $C^{2}$ des courbes de choc et de détente (voir [12] et [9] pour le cadre général). Si on introduit la relation (27) dans l'inégalité d'entropie :

$$
-\sigma[\eta]+[U \eta]<0
$$

on obtient : $[U]<0$, et la paramétrisation des chocs $(\beta=(\gamma+1) /(\gamma-1))$,

$$
\frac{\rho_{r}}{\rho_{1}}=z, \quad \frac{P_{r}}{P_{1}}=\frac{\beta z-1}{\beta-z}, \quad \frac{K_{r}}{K_{1}}=\frac{4 z-1}{4-z}, \quad \frac{\varepsilon_{r}}{\varepsilon_{1}}=\frac{\mu z-1}{\mu-z} .
$$

$$
\frac{u_{r}-u_{1}}{c_{1}}= \pm \frac{z-1}{z^{1 / 2}}\left(\frac{2}{(\gamma-1)(\beta-z)}+\frac{10 K_{1}}{3 \gamma P_{1}(4-z)}\right)^{1 / 2}
$$

( $\left.\mu=\left(C_{\varepsilon 1}+3\right) / C_{\varepsilon t}\right)$, avec un signe négatif pour le 1-choc $(z>1)$ et positif pour le 5-choc $(0<z<1)$ dans la formule (31). A travers la discontinuité de contact, on a :

$$
\sigma=u_{r}=u_{1}, \quad P_{r}-P_{1}+\frac{2}{3}\left(K_{r}-K_{1}\right)=0 .
$$




\section{A. Forestier, J.-M. Hérard et X. Louis}

On peut donc s'intéresser au problème de Riemann associé aux conditions initiales :

$$
\begin{aligned}
& \mathbf{W}(x<0, t=0)=\mathbf{W}_{\ell}=\left(\rho_{L}, \rho_{L} U_{L}, E_{L}, K_{L}, \varepsilon_{L}\right), \\
& \mathbf{W}(x>0, t=0)=\mathbf{W}_{\ell}=\left(\rho_{R}, \rho_{R} U_{R}, E_{R}, K_{R}, \varepsilon_{R}\right) .
\end{aligned}
$$

Proposition IV. - Le problème de Riemann unidimensionnel associé au système non conservatif (17)-(21) et aux relations de saut approchées (27)-(28) admet une unique solution entropique à densité strictement positive si, et seulement si.

$$
U_{R}-U_{L}<X_{L}+X_{R},
$$

en posant:

$$
X_{i}=\int_{0}^{\rho_{1}} \frac{c^{\prime}\left(\rho, s, K \rho^{-5 / 3}\right)}{\rho} d \rho \quad \text { avec } i=L \text { ous } R .
$$

La pression $P$ et l'énergie cinétique turbulente $K$ restent alors positives dans le plan $(x, t)$.

La preuve (voir [12] et [13]) s'obtient par simple étude de fonctions; la résolution pratique du problème de Riemann nécessite néanmoins la résolution d'un système de deux équations non linéaires $(2 \times 2)$ (voir [12]). On obtient évidemment le résultat classique de la DG lorsque l'on pose formellement : $K=0$. La paramétrisation de la courbe de pression dans (30) est identique à celle de la DG. Si l'on * oublie " le terme non conservatif dans l'équation (14), l'étude du système conservatif (qui est encore hyperbolique), montre que la pression peut devenir négative dans les détentes (voir [7] et [12]), ce qui interdit l'utilisation « numérique * de la solution du problème de Riemann associé. L'examen de la préservation de la positivité des variables $\rho, P$ et $K$ dans le cadre de solutions régulières est abordé dans [13] (voir également [15] et [16]).

\section{Un schéma de type Godunov (voir [17])}

Le système convectif non conservatif unidimensionnel (17)-(21) peut s'écrire comme suit :

$$
\begin{gathered}
\mathrm{W}_{, \ell}+\left(\mathrm{F}_{x}(\mathrm{~W})\right)_{, s}+\mathrm{B}(\mathrm{W}) \mathrm{W}_{, x}=0 \\
\left(\mathrm{~F}_{x}(\mathrm{~W})\right)^{\ell}=\left(\rho U, \rho U^{2}+P+\frac{2}{3} K, U\left(E+P+\frac{2}{3} K\right), U K, U \varepsilon\right) \\
\left(\mathrm{B}(\mathrm{W}) \mathbf{W}_{. x}\right)^{\ell}=\left(0,0,0, \frac{2 K}{3} U_{, s}, \frac{2 \varepsilon}{3} C_{e_{1}} U_{, x}\right) .
\end{gathered}
$$

On sait que la simulation numérique de systèmes hyperboliques non conservatifs est très délicate dans le cas de chocs forts, même si ceux-ci dérivent de lois de consenvation bien identifiées ([18]). On pourrait a priori envisager, dans un cadre unidimensionnel, de considérer le résultat de la projection $L^{2}$ des deux demi-solutions des problèmes de Riemann associés aux interfaces $(i \pm 1 / 2)$, soit :

$$
\begin{aligned}
W_{i}^{n+1} h_{i}= & \int_{x_{i-1 / 2}}^{x_{1}} W^{\text {Riemann }}\left(W_{1-1}^{n}, W_{1}^{n}, \frac{x-x_{i-1 / 2}}{\Delta t}\right) d x \\
& +\int_{s_{1}}^{s_{1+1 / 2}} W^{\text {Riemann }}\left(W_{1}^{n}, W_{1+1}^{n}, \frac{x-x_{i+1 / 2}}{\Delta t}\right) d x
\end{aligned}
$$


Ce schéma préserve les propriétés de positivité, mais il s'avère délicat de l'étendre au cadre multidimensionnel sur maillage non structuré. On propose le schéma explicite d'intégration :

$$
\begin{gathered}
\left(\mathbf{W}_{\mathrm{z}}^{n+1}-\mathrm{W}_{\mathrm{n}}^{n}\right) h_{i}+\Delta t\left(\mathbb{F}_{x}\left(\mathbf{W}_{t+1 / 2}^{\mathrm{God}}\right)-\mathbb{F}_{x}\left(\mathbf{W}_{t-1 / 2}^{\mathrm{God}}\right)\right)+\Delta t \mathbf{X}_{2}=0 \\
\mathbf{X}_{\mathrm{t}}^{\mathrm{t}}=\left(0,0,0, \frac{2}{3} \hat{K}_{i}\left(U_{\imath+1 / 2}^{\mathrm{God}}-U_{i-1 / 2}^{\mathrm{God}}\right), \frac{2}{3} C_{\varepsilon_{3}} \hat{\varepsilon}_{1}\left(U_{i+1 / 2}^{\mathrm{God}}-U_{t-1 / 2}^{\mathrm{God}}\right)\right)=0,
\end{gathered}
$$

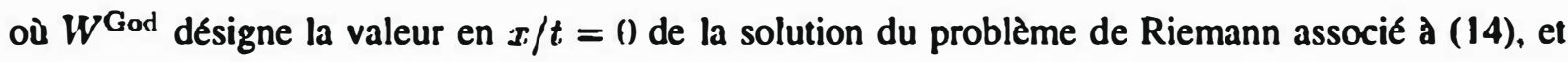

$$
(\hat{K})_{i}^{n}=\left(K_{i+1 / 2}^{\mathrm{God}}+K_{i-1 / 2}^{\mathrm{God}}\right) / 2, \quad(\hat{\varepsilon})_{i}^{n}=\left(\epsilon_{i+1 / 2}^{\mathrm{God}}+\epsilon_{i-1 / 2}^{\mathrm{God}}\right) / 2
$$

Le choix (39) permet de retrouver formellement le schéma conservatif de Godunov, pour l'équation de Burgers récrite sous forme non conservative. La figure 1 illustre le comportement numérique de variable pression moyenne $(P+2 K / 3)$, pour le probleme de Riemann associé aux valeurs initiales $\left(\rho_{L}=1\right.$., $\left.U_{L}=0 ., P_{L}=100000, K_{L}=100\right)$ et $\left(\rho_{R}=0.125, U_{R}=0 ., P_{R}=10000, K_{R}=1(100)\right.$. On observe notamment sur ce cas une nette variation de $P$ à travers la discontinuité de contact, alors que $(P+2 K / 3)$ reste numériquement invariant dans cette zone (ainsi que $U$, voir (32)).

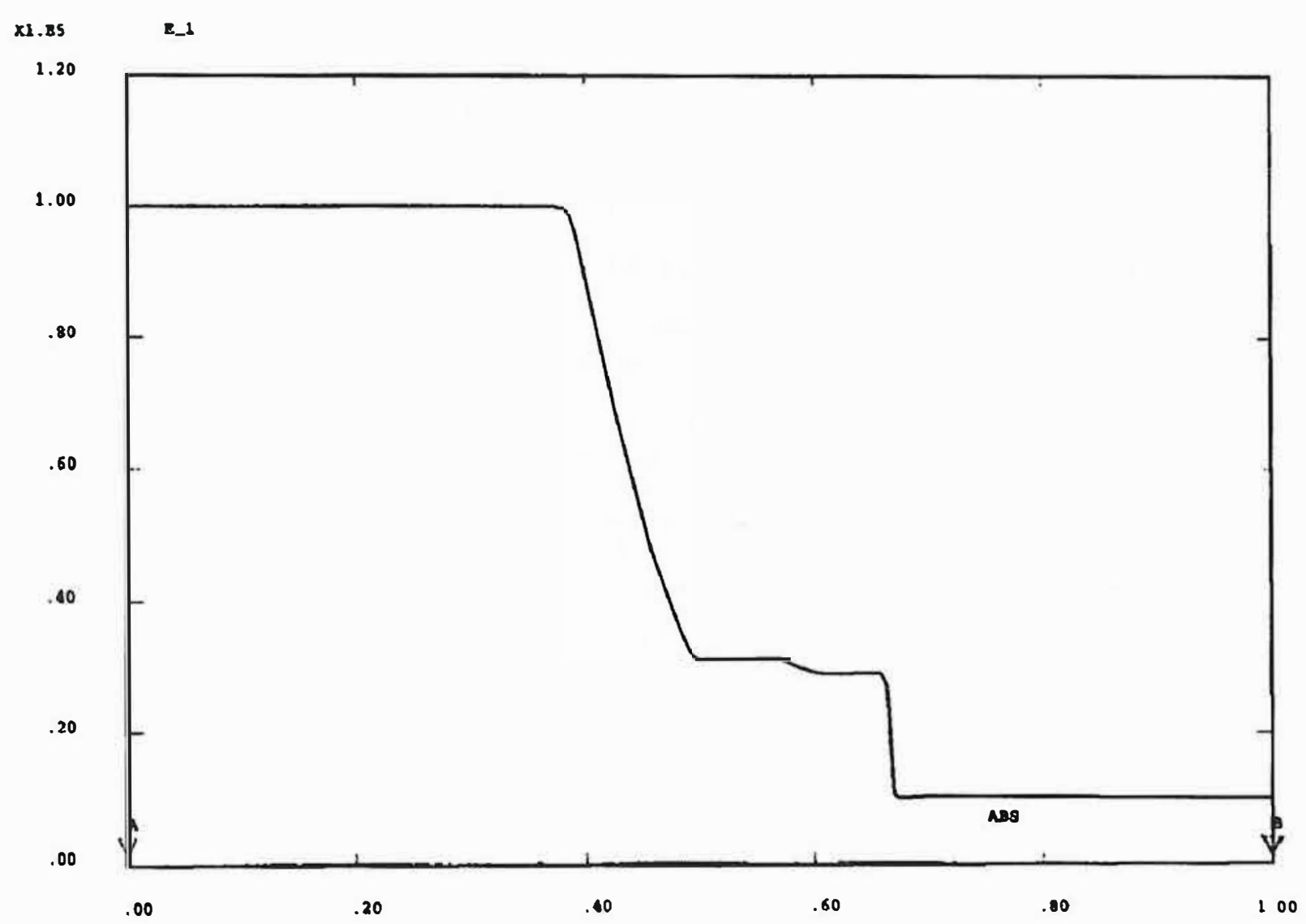

Fig. 1. - Mean pressure $P$ (Shock tube experiment).

Fig. 1. - Pression movenne (rube a choc). 


\section{A. Forestier, J.-M. Hérard et $X$. Louis}

\section{Conclusion}

La principale originalité du travail présenté réside dans l'examen du système de convection complet qui est non conservatif. Le solveur de type Godunov proposé peut s'implémenter dans un cadre multidimensionnel sur maillage non structuré (voir [12]). Les résultats numériques obtenus dans le cadre de simulations de problèmes de Riemann unidimensionnels confirment les qualités du solveur explicite, lorsque les amplitudes de choc sont faibles. Un solveur de Riemann approché de type Roe a également été développé (voir [7]), qui donne des résultats satisfaisants dans le cas de chocs faibles.

Remerciements. Les auteurs remercient Yvon MADAY pour les conseils et encouragements prodigués.

Note remise le 22 novembre 1996, acceptée le 16 décembre 1996.

\section{Références bibliographiques}

[II D. Vandromme et II. Ila Mlinh, 1986. About the coupling of turbulence closure models with averaged Navier-Stokes equations, J.C.P., Vol. 65, n 2 . pp. 386-409.

|2| B. Cardot, B. Coron, B. Mohammadi et O. Pironneau, 1991. Simulation of turbulence with the $k-\varepsilon$ model. Comput. Meshods Appl. Mech. Eng.. vol. 87, pp. 103-116.

(3) B. Mohammadi et O. Pironneau, 1994. Analysis of the $k-\varepsilon$ surbulence mexdel, Masson/Wiley.

14) A. Fovre, L. S. G. Kovasnay, R. Dumas, J. Gaviglio et M. Coantic, 1976. La turhulence en méranique des fluides. Gauthier-Villars.

|5| K. Jansen, 2. Johan, T. J. R. Hughes, 1993. "Implementation of a one equation turbulence model within a stabilized tinite element fonnulation of a symmetric advective diffusive system". Comput. Mlethrads Appl. Mech. Engrg., vol. 105. pp. $405-433$.

|6| C. Olivier et B. Larrouturou, 1991. "On the numerical approximation of the $K-\varepsilon$ turbulence model for two dimensional compressible flows". INRIA reporn, 1526.

[7] J. M. Hérard, 1995. "Solveur de Riemann approché pour un système hyperbolique non conservatif issu de la turbulence compressible". rappon EDF/DER HE-41/95/009/A.

[8] J. F. Colombeau, 1992. "Mulsiplication of distriburions". Springer Verlag.

|9| P. Le Floch, 1988. "Entropy weak solutions to non linear hyperbolic systems in non conservative forn", Comm. Partiul Differential Equations, 1316). pp. 669-727.

[10| P. Le Floch et T. P. Liu, 1992. "Existence theory for non linear hyperbolic systems in non conservative form", C.M.A.P. report $n^{\circ} 254$. Ecole Polytechnique. France.

[1I] L. Sainsaulieu, 1995. Thèse d'habilitation. Université Paris VI. 2001/1995.

[12] X. Louis, 1995. Thèse de doctorat . Université Paris VI. 06/07/1995.

|13| J. M. Ilérard, A. Forestier, X. Louis, 1994. "Un système non strictement hyperbolique de description de la turbulence compressible". rappon EDF/DER HE-4I/94/01//A. 1994.

$[14]$ J. Smoller, 1982. "Shock waves and Reacrion-Diffusion Equations". Springer Verlag.

[ISI R. Lewandowskl, 1993. * Modeles de turbulence el ćquatıons paraboliques * C. R. Acau. Sci. Paris Ser. 1. 317, pp. 835-840.

|16| R. Lewandowskl, 199\&. * Les équations de Stokes et Navier-Stokes couplées avec l'équation de l'énergie cinétique turbulente ^ C. R. Acad. Sri. Paris Ser. 1. 318. pp. 1097-1102.

II7| S. K. Godunov, 1959. * A difference scheme for numerical computation of discontinuous solutions of equations of fluid dynamics $\%$. Math Sh., 47(89), pp. 271-306.

[18] F. De Vuyst, 1994. Thèse de Ductorat, Universite Paris VI , 19/12/1994. 